\title{
Polydimethylsiloxane- $\mathrm{LiNbO}_{3}$ surface acoustic wave micropump devices for fluid control into microchannels
}

\author{
Salvatore Girardo, ${ }^{a}$ Marco Cecchini, ${ }^{b}$ Fabio Beltram, ${ }^{b}{ }_{\text {Roberto Cingolani }}{ }^{a}$ and Dario Pisignano ${ }^{a}$ \\ Received 7th March 2008, Accepted 3rd June 2008 \\ First published as an Advance Article on the web 30th July 2008 \\ DOI: 10.1039/b803967d
}

This paper presents prototypical microfluidic devices made by hybrid microchannels based on piezoelectric $\mathrm{LiNbO}_{3}$ and polydimethylsiloxane. This system enables withdrawing micropumping by acoustic radiation in microchannels. The withdrawing configuration, integrated on chip, is here quantitatively investigated for the first time, and found to be related to the formation and coalescence dynamics of droplets within the microchannel, primed by surface acoustic waves. The growth dynamics of droplets is governed by the water diffusion on $\mathrm{LiNbO}_{3}$, determining the advancement of the fluid front. Observed velocities are up to $2.6 \mathrm{~mm} \mathrm{~s}^{-1}$ for $30 \mathrm{dBm}$ signals applied to the interdigital transducer, corresponding to tens of $\mathrm{nl} \mathrm{s}^{-1}$, and the micropumping dynamics is described by a model taking into account an acoustic power exponentially decaying upon travelling along the microchannel. This straighforward and flexible micropumping approach is particularly promising for the withdrawing of liquids in lab-on-chip devices performing cycling transport of fluids and biochemical reactions.

\section{Introduction}

The demand for low cost and highly portable devices for biochemical applications has greatly stimulated the development of micro-total analysis systems ( $\mu \mathrm{TAS}$ ) and of microfluidic science and technology. ${ }^{1}$ The miniaturization of these devices led to better performance with respect to traditional analytical methods, since it involved smaller quantities of samples and reagents, allowing more reactions to occur in parallel on the same chip, more quickly and effectively, and with reduced manual intervention.

For a full exploitation of the advantages of microfluidics, one needs highly controlled liquid flows, which can be achieved by passive encoding of capillarity relying on appropriate flow resistances, ${ }^{2}$ or by external or integrated actuation. ${ }^{3}$ Aqueous solutions and other polar fluids can fill hydrophilic microchannels by spontaneous capillarity, but this motion is often not precisely controlled and hard to be used for most analytical applications. In the common case of hydrophobic capillaries, polar fluids must be forced into microchannels by means of active pumping elements, overcoming the large resistance to flow due to small microchannel sections. Following the demonstration of the first piezoactuated silicon-based planar micropump ${ }^{4}$ different classes of active elements have been proposed for microfluidics, including devices able to generate continuous liquid flows. Reciprocating displacement microp-

${ }^{a} N N L$, National Nanotechnology Laboratory of CNR-INFM, Università del Salento c/o Distretto Tecnologico ISUFI, and Italian Institute of

Technology, via Arnesano, I-73100, Lecce, Italy.

E-mail: Dario.pisignano@unile.it; Tel: +390832298146

${ }^{b}$ Scuola Normale Superiore, NEST-CNR-INFM and Italian Institute of

Technology, Piazza dei Cavalieri 7, I-56126, Pisa, Italy.

E-mail: m.cecchini@sns.it; Tel: +39050509459 umps, in which the pressure work is carried out by the movement of flexible diaphragm surfaces in the device chamber, exploit piezoelectric, ${ }^{5}$ thermopneumatic, ${ }^{6}$ pneumatic, ${ }^{7}$ or electrostatic ${ }^{8}$ actuation, and exhibit typically reported maximum flow rates from fractions to almost $10^{3} \mu 1 \mathrm{~s}^{-1}{ }^{3}$ These pumps require optimal sealing between the device layers, and generally produce the driving pressure outside the chips, thus needing specific fluidic connections, reducing the portability of the overall $\mu$ TAS architecture and leading to large volumes of analyte waste in the tubing networks. Dynamic, continuous flow micropumps are instead based on the interaction of fluids with an electromagnetic field through electro- ${ }^{9}$ and magneto-hydrodynamic, ${ }^{10}$ electroosmotic, ${ }^{11}$ electrowetting, ${ }^{12}$ or electrochemical ${ }^{13}$ methods. They can be generally integrated without invasive external connections, and have been reported to generate steady-state flow rates up to the order of $10^{2} \mu \mathrm{s} \mathrm{s}^{-1} .^{3}$ Unfortunately, electrohydrodynamic pumps require high voltages $(400-6000 \mathrm{~V}),{ }^{3}$ and they are very sensitive to the properties of employed fluids (such as ionic strength and composition, $\mathrm{pH}$, and permittivity) and to the presence of electrical charges on the microchannel surfaces. Recently, methods for all-optical control of microflows have also been suggested, although still relying on external lightemitting sources for excitation..$^{14,15}$ Other difficulties are related to fundamental requirements for the practical use of devices, i.e. cheapness, straightforward packaging, and ease of interfacing with control units.

In the last years, the interaction between surface acoustic waves (SAWs) and liquids began to be studied as a pumping approach, relying on the streaming effect that drives the fluid flow in the direction of SAW propagation. ${ }^{16-19}$ Kurosawa et al. developed the first device for liquid atomization by SAW, ${ }^{16}$ and different platforms were implemented for handling, moving, splitting, mixing and merging microdroplets deposited 
on surfaces. ${ }^{18-21}$ Recently, acoustic streaming was employed to generate flows with high shear rates $\left(>10^{3} \mathrm{~s}^{-1}\right)$ and mimic blood conditions for the investigation of circulatory proteins, ${ }^{22}$ and for collecting and concentrating airborne microparticles for environmental monitoring applications. ${ }^{23}$ The broad interest towards acousto-fluidic interactions has also led to in-depth investigations by micron-resolution particle image velocimetry and finite element numerical simulations. ${ }^{24}$ The streaming occurs because of the pressure gradient generated by the dissipation of the acoustic energy inside fluid elements standing on a piezoelectric substrate. However, so far SAW methods have been mainly limited to mixing, localization or transport of droplets deposited on planar substrates, preferably patterned by regions of different wettability. The main issues of such open digitalized microfluidic architectures are the liquid evaporation and a remarkable sensitivity to surface contamination. However, closed microchannel environments allow one to incubate and store fluid assays over long times, and to directly control and measure the liquid volumes through the confined channel geometries. In addition, a limitation of previously reported SAW-based approaches stands in the fact that only forward actuation was observed by acoustic streaming, i.e. the acoustic pressure gradient generating the flow is along the direction of wave propagation, thus being suitable for forward injection micropumping only. Many lab-on-a-chip devices and applications need simple methods for withdrawing in order to recover fluids and to better control the liquid displacement among cycling reactions.

In this work, we investigate the application of the SAWbased pumping method to microchannel environments fully compatible with $\mu$ TAS applications, studying the flow of water in prototypical devices made by a piezoelectric lithium niobate $\left(\mathrm{LiNbO}_{3}\right)$ substrate and elastomeric polymer patterns defining the capillary circuits. We find that this architecture enables withdrawing control of microflow by means of a single interdigital transducer (IDT), producing flow rates were up to many tens of $\mathrm{nl} \mathrm{s}^{-1}$. The device operation is found to be related to the SAW-induced formation and coalescence of droplets within the microchannel.

\section{Experimental}

Our devices are fabricated by the contact superposition of two layers defining the microchannel geometry. The bottom layer is a $\mathrm{LiNbO}_{3}$ piezoelectric substrate, with microfabricated IDTs for SAW excitation and detection. Some typical IDT structures, fabricated by photolithography, metal evaporation and subsequent lift-off, are displayed in Fig. 1a and b. For the pumping experiments, the IDT chips were accomodated in a homemade chip holder, equipped by a Peltier refrigerator coupled with a Thorlabs TED350 controller (Fig. 1c). While the Peltier element allowed us to stabilise the chip temperature at $T=19{ }^{\circ} \mathrm{C}$ in the here reported experiments, no significant difference was observed in the device operation also well above room temperature. The IDTs employed for the present experiments are composed by 20 pairs of $500 \mu \mathrm{m}$-long $\mathrm{Al}$ fingers with periodicity $(2 d)$ of $24 \mu \mathrm{m}$, and generate acoustic waves on the piezoelectric surface with wavelength, $\lambda=2 d$. This corresponds to a resonance frequency, $f_{0}=v / \lambda$, of about
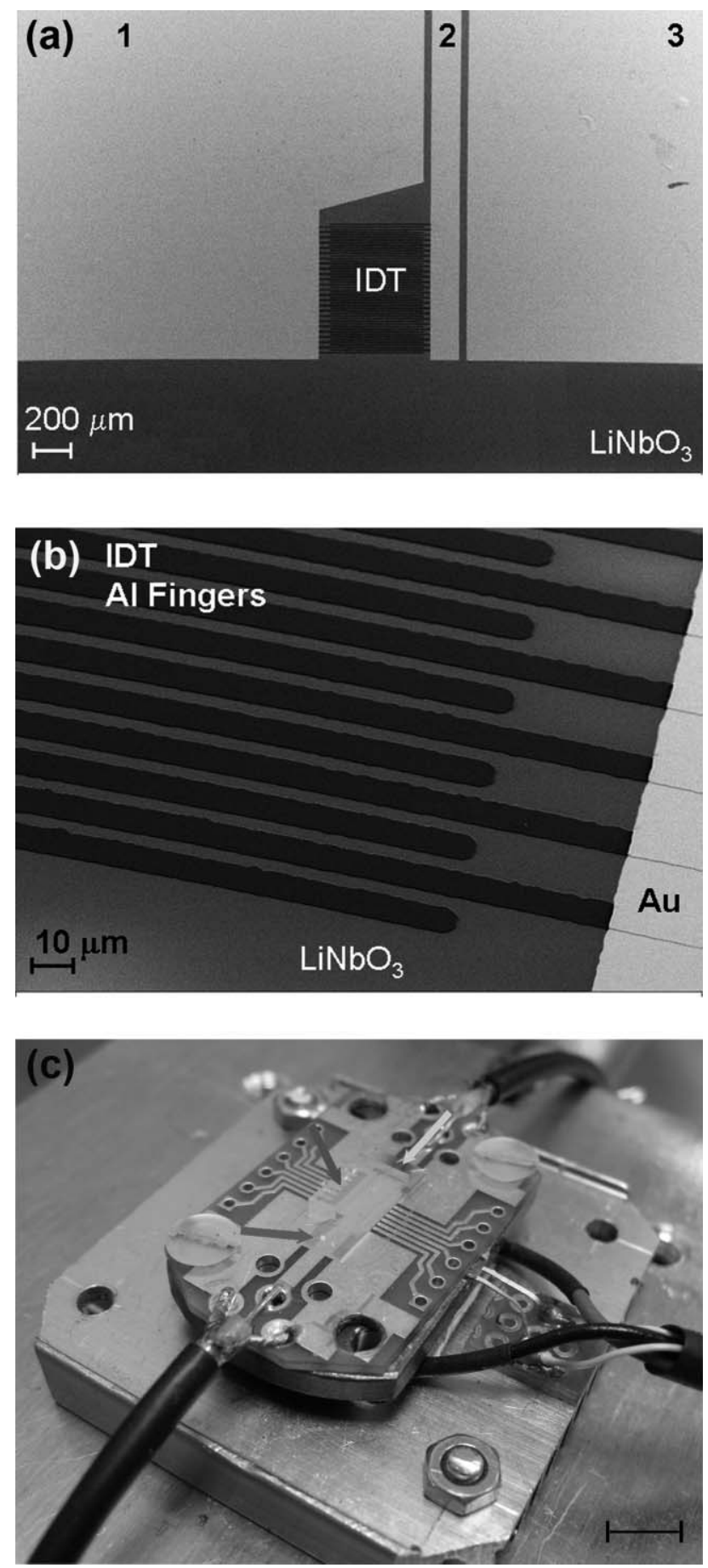

Fig. 1 (a) Low-magnification scanning electron microscopy image of an IDT: 1, 2, 3 are the coplanar waveguide gold electrodes connecting the IDT to the off-chip wiring. (b) Detail of the IDT aluminum fingers. (c) Photograph of the chip mounted in our homemade holder providing temperature control and electrical connections for SAW excitation, with a water drop released and lying at the chip entrance without penetrating into microchannels. The left, middle, and right arrows indicate the positions of the external water reservoir, of the hybrid microchannel, and of the IDT, respectively. Marker $=5 \mathrm{~mm}$.

$160 \mathrm{MHz}$ on $\mathrm{LiNbO}_{3}$ (Fig. 2). In the previous expression, $v$ indicates the wave propagation speed. 


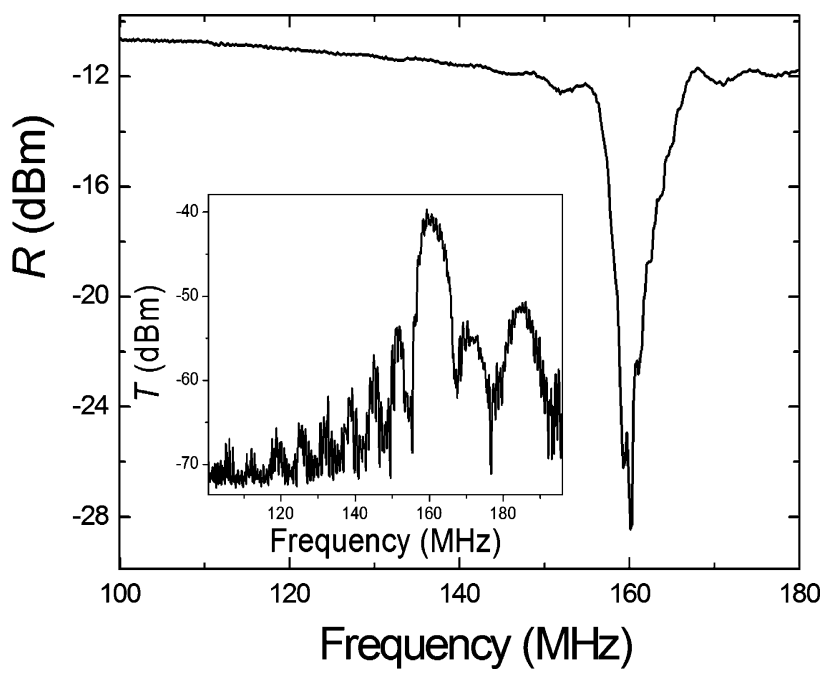

Fig. 2 Radio-frequency (RF) power reflected by an IDT as a function of the RF excitation frequency. Inset: transmission characteristic of a delay line composed by two IDTs placed at a distance of $3.4 \mathrm{~mm}$. Excitation power $=0 \mathrm{dBm}$.

The upper layer of our devices is a polydimethylsiloxane (PDMS) element with a textured microchannel realized by replica molding. ${ }^{25}$ The master structures employed for templating the PDMS replicas were fabricated by either (i) photolithography and subsequent etching on $\mathrm{Si} / \mathrm{SiO}_{2}$ substrates or (ii) photolithography and curing of the negative, high glass transition temperature photoresist SU8. In the first procedure, after the deposition of photoresist (Clariant AZ5214E :AZ EBR Solvent $1: 1)$ on $\mathrm{Si} / \mathrm{SiO}_{2}$ wafers, UV-exposure $\left(8^{\prime} 30^{\prime \prime}\right.$ at $\left.45 \mathrm{~W}\right)$ and development of the resist, the thermal dioxide was etched by means of $\mathrm{NH}_{4} \mathrm{~F} / \mathrm{HF} / \mathrm{H}_{2} \mathrm{O}(6.6 \mathrm{~g} / 1.6 \mathrm{ml} / 10 \mathrm{ml})$. The so obtained $\mathrm{SiO}_{2}$ mask was then employed to etch the underlying silicon by $5.9 \mathrm{M}$ solutions of $\mathrm{KOH}$ at $80{ }^{\circ} \mathrm{C}$. In the second procedure, masters with patterned relief structures were made directly by SU-8 (MicroChem Corp., Newton, MA) negative photoresist, spin-cast on $\mathrm{Si}-\mathrm{SiO}_{2}$ substrates and cured for $15^{\prime}$ at $150{ }^{\circ} \mathrm{C}$ after exposure. Resist thicknesses between 20 and $50 \mu \mathrm{m}$ were obtained by properly tailoring the developer : SU-8 (2100 grade) relative concentration $(>5: 1)$. The elastomeric replicas were then realized by in situ polymerization $\left(140{ }^{\circ} \mathrm{C}, 15^{\prime}\right)$ of PDMS (Sylgard 184 by Dow Corning, Midland, MI, A : B 1 : 9) on the $\mathrm{Si} / \mathrm{SiO}_{2}$ or on the SU8 masters.

PDMS microchannels with lateral dimensions between 210 and $520 \mu \mathrm{m}$ were then aligned orthogonally to the IDT fingers and deposited on $\mathrm{LiNbO}_{3}$ to assemble the devices (Fig. 3). The alignment can be performed manually, or by an aligner equipment (EVG 620) for layer-by-layer lithography with soft stamps. ${ }^{26}$ Differently from previous experiments, ${ }^{18,19,21}$ no functionalization, unfouling, or glueing layer were deposited on the piezoelectric surface.

To test the system, we released a deionised water drop of about $2 \mu \mathrm{l}$ at the edge of the microchannels from a micropipette, and applied to the IDT a signal with power $(W)$ comprised between 20 and $30 \mathrm{dBm}$, whose values where chosen in order to observe SAW-induced liquid motion without causing device damages, which could occur because of the high electrochemicalcoupling coefficient of $\mathrm{LiNbO}_{3}$. The water static contact angles

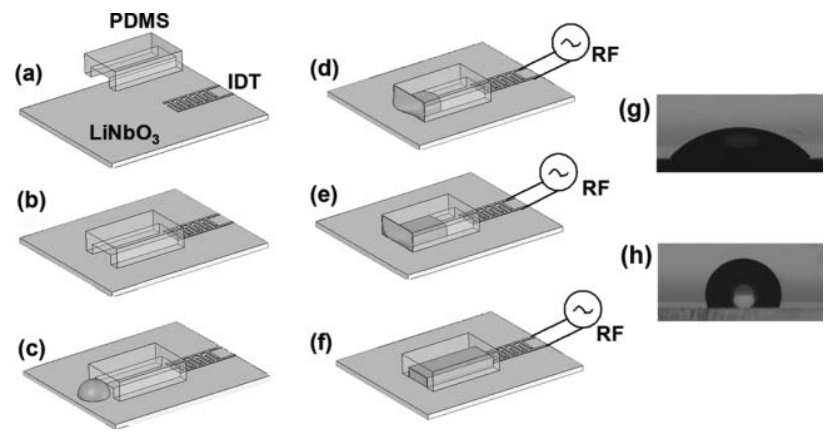

Fig. 3 Scheme of device assembling (a)-(b), $2 \mu 1$ drop release (c), and withdrawing operation (d)-(f). RF = exciting radiofrequency. (g), (h): Photographs of the contact angle, $\theta$, for $3 \mu \mathrm{l}$ drops of water on $\mathrm{LiNbO}_{3}$ $(\mathrm{g}), \theta_{\mathrm{LiNb}_{3}}=50 \pm 4^{\circ}$, and on a PDMS surface (h), $\theta_{\mathrm{PDMS}}=117 \pm 4^{\circ}$.

on PDMS and $\mathrm{LiNbO}_{3}$ were measured by an Optical Video Contact Angle System CAM-200 KSV. A drop of distilled water $(\approx 3 \mu \mathrm{l})$ was delivered onto the investigated surfaces by a capillary tip mounted on a one-touch mechanical dispenser. Pictures of the drop were recorded by a camera collecting 30 frames per second with a resolution of $512 \times 480$ pixels, illuminating the drop samples by a blue light-emitting diode.

The filling rate during SAW pumping was observed by optical microscopy through a lateral ruler integrated in the chip, having parallel ticks separated by $100 \mu \mathrm{m}$. A Leica MZ16 stereomicroscope coupled to a Basler A602f-2 fast acquisition camera (400 fps at $640 \times 120$ pixel resolution), with a direct hard-disk writing system for long-time recording (Advanced Technologies), were employed to determine the exact position $(z)$ of the fluid front along the microchannels during the entire pumping process. We could not observe any appreciable contamination or liquid leakages during filling of the microchannels, which allowed us to replace only the polymeric stamp and to re-use the same piezoelectric substrate for many experiments.

The suitability of the method to pump biological samples was tested by loading $2 \mu \mathrm{l}$ of $\mathrm{E}^{1} \mathrm{GFP}$ fluorescent protein ${ }^{27}$ aqueous solution $(40 \mu \mathrm{M}$, buffered at $\mathrm{pH}=7.5)$ into the devices. The protein fluorescence was excited by a $488 \mathrm{~nm}$ laser, and collected by a charge-coupled-device camera after filtering the emission with a z4881p filter (CHROMA Technology Corp.).

\section{Results and discussion}

Upon releasing water droplets at the edge of opened microchannels, the overall balance of the liquid-solid interfacial tensions disfavours the spontaneous penetration of the liquid (contact angle photographs in Fig. $3 \mathrm{~g}$ and $\mathrm{h}$ ), ${ }^{28}$ and the capillaries are not filled by the fluid.

After switching on the SAW actuation with droplets positioned between the IDT and the channel entrance, we observe first the deformation of the liquid surface by acoustic streaming, in agreement with previous reports. ${ }^{17,18,20}$ For excitation power larger than $20 \mathrm{dBm}$, the drops are forced to penetrate within the microchannels with typical filling velocities of the order of tens of $\mu \mathrm{m} \mathrm{s}^{-1}$. The relevant liquid atomization external to the microchannel can lead to significant evaporation and results in an amount of water of the order of $10^{-1}-1 \mathrm{nl}$ pumped into the capillary. 
Instead, exciting the IDT with droplets positioned on the opposite microchannel entrance with respect to the PDMS element, as depicted in Fig. 3, surprisingly leads to very effective flow of water in the microchannel, i.e. to moving liquids in the opposite direction with respect to the wave propagation. ${ }^{29}$ This effect is relevant since it would allow one to withdraw the flow within microfluidic devices, through a proper choice of the capillary geometries. The withdrawn filling dynamics is driven by the formation of liquid particles condensing within the microchannel in front of the penetrating liquid interface (Fig. 4). In particular, we observed the liquid atomization in the microchannel, leading to the formation of droplets with typical radius $\left(r_{\mathrm{d}}\right)$ below $12 \mu \mathrm{m}$ (Fig. 5), which is followed by either streaming of the water particles towards the main liquid front (namely travelling along the SAW propagation direction) or reevaporation within the restricted capillary environment. During the SAW actuation, the initial population of droplets initially evolves towards an increase of the average values of $r_{\mathrm{d}}$, such as in the frames (a)-(f) of Fig. 4. Afterwards, the main liquid front starts moving in the microchannel, the overall droplet radii distribution remaining largely stationary over time (Fig. 4h1), with a full width at half maximum of about $1.5 \mu \mathrm{m}$. The continuous coalescence of the droplets with the liquid front progressively determines the advancement of the contact line.
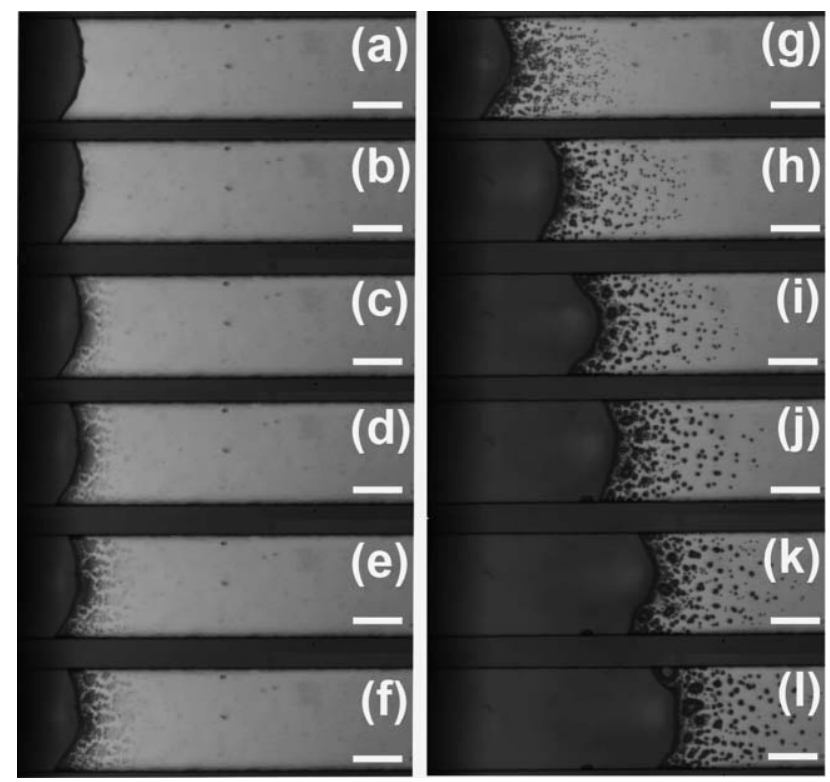

Fig. 4 Photographs of the water withdrawing micropumping at different times $(t)$. From (a) to (1): $t=0.00,0.05,0.06,0.08,0.09,0.13,0.53$, $1.51,2.46,3.48,4.49$, and $5.49 \mathrm{~s}$, respectively. Marker $=100 \mu \mathrm{m}$.

The time dependence of the radius of individual droplets induced by SAW up to coalescence (mutual or with the advancing water front), is mainly diffusion-limited (Fig. 6), similarly to previously reported optofluidic experiments. ${ }^{14}$ In fact, the impingement of water molecules after adsorption and diffusion on the hydrophilic bottom wall of the microchannels determines a droplet growth dynamics well described by a law: $r_{\mathrm{d}}=A t^{1 / 4},{ }^{30}$ where $A$ is a constant depending on the contact angle, the number density of water, and the saturated concentration of water molecules on the $\mathrm{LiNbO}_{3}$ surface (fitting lines in Fig. 6). Differently, the merging of neighbouring water

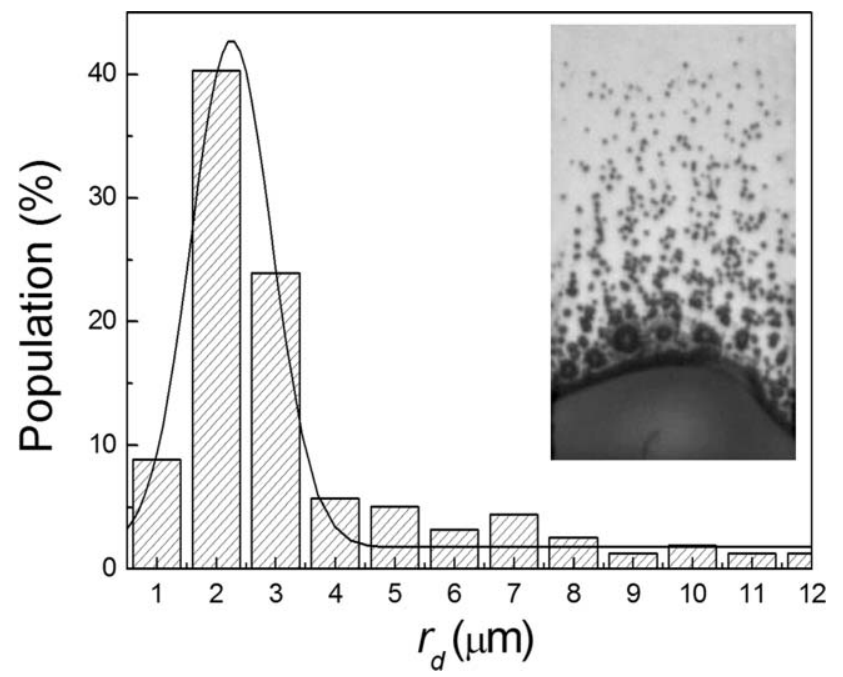

Fig. 5 Obtained radius $\left(r_{\mathrm{d}}\right)$ distribution of the droplet population generated by SAW actuation in the microchannel during withdrawing micropumping. The maximum observed droplet radius $(\cong 12 \mu \mathrm{m})$ roughly corresponds to half SAW wavelength. The superimposed line is the best fit to experimental data by a Gaussian curve. Inset: micrograph of a typical droplets population $(194 \mu \mathrm{m} \times 346 \mu \mathrm{m})$. The advancing water/ $\mathrm{LiNbO}_{3}$ contact line is visible in the bottom part of the picture.

particles determines a much faster increase of the droplet radii, as evidenced in Fig. 6 by the experimental data collected in correspondence to mutual coalescence $(t>2 \mathrm{~s}) .^{30}$

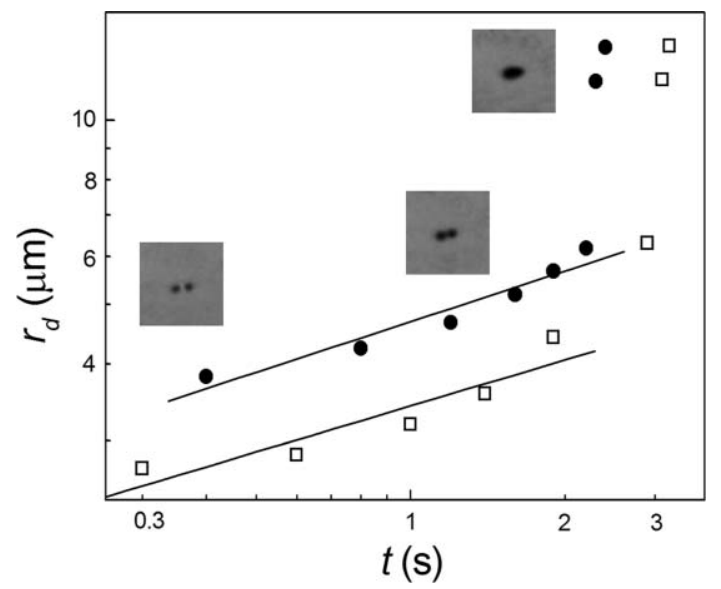

Fig. $6 \quad r_{\mathrm{d}} \quad v s$ t for two droplets (circles and squares) during SAW withdrawing in the microchannel. The sudden increase of the $\left(r_{\mathrm{d}}, t\right)$ slope after about $2 \mathrm{~s}$ is due to coalescence with neighboring droplets. The superimposed lines are the best fits to the experimental growth dynamics by a $r_{\mathrm{d}} \propto t^{1 / 4}$ law before coalescence, indicating the predominance of diffusion-limited mechanisms. Inset: micrographs $(57 \mu \mathrm{m} \times 57 \mu \mathrm{m})$ of two neighboring droplets during formation (left), growth (middle), and coalescence (right).

The typical $(z, t)$ curves for SAW withdrawing by different values of the power of the signal applied to the IDTs are displayed in Fig. 7, showing a rapid microchannel filling, with a monotonous increase of the microflow rate upon water penetrating in the capillaries, as expected since this drives the fluid closer to the SAW source region. For $W=27 \mathrm{dBm}$, it takes a time $t_{1}=370 \mathrm{~s}$ for water to completely fill a $5.2 \mathrm{~mm}$ 

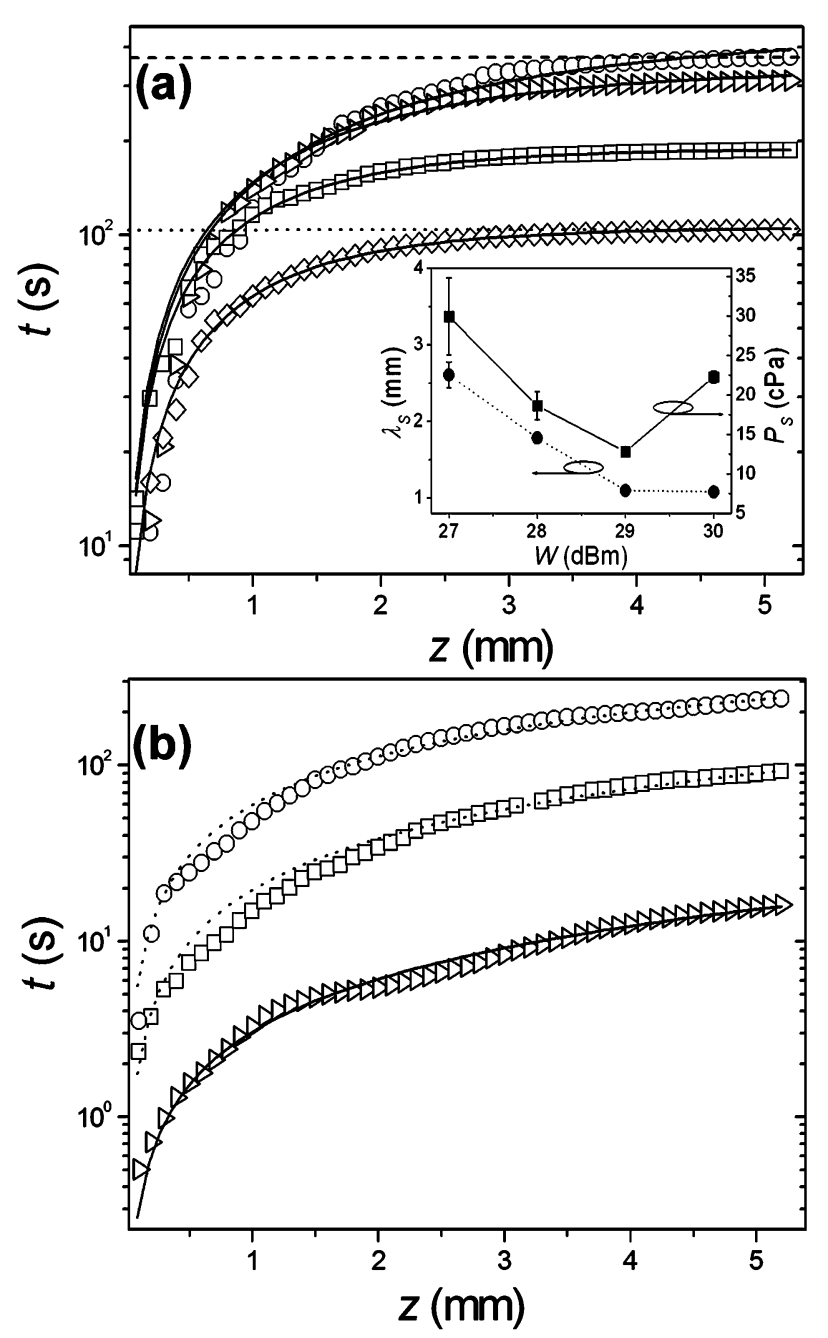

Fig. 7 (a) Experimental $(z, t)$ curves describing SAW-actuated withdrawing micropumping in a channel with section $210 \times 20 \mu \mathrm{m}^{2}$, for different signals applied to the IDTs. $W=27 \mathrm{dBm}(\bigcirc), 28 \mathrm{dBm}(\triangleright)$, $29 \mathrm{dBm}(\square)$, and $30 \mathrm{dBm}(\diamond)$. Superimposed lines: best fits to experimental data according to eqn (2). Inset: SAW pressure decay length in the device, $\lambda_{\mathrm{S}}$ (circles, left vertical scale) and pressure, $P_{\mathrm{S}}$ (squares, right vertical scale) $v s W$. The horizontal lines indicate the time for complete filling of our microchannels by $27 \mathrm{dBm}\left(t_{1}\right.$, dashed line) and $30 \mathrm{dBm}\left(t_{2}\right.$, dotted line). (b) Experimental $(z, t)$ curves of SAW-actuated withdrawing in a channel with section $520 \times 50 \mu \mathrm{m}^{2}$, for $W=25 \mathrm{dBm}(\bigcirc), 26 \mathrm{dBm}(\square)$, and $28 \mathrm{dBm}(\triangleright)$. The line superimposed to the $25 \mathrm{dBm}$ data is the best fit according to eqn (2).

long channel with section $210 \times 20 \mu \mathrm{m}^{2}$ (Fig. 7a). Increasing the excitation power to $30 \mathrm{dBm}$, the filling time drops down to $t_{2}=103 \mathrm{~s}$. The maximum water penetration velocities range from $120 \mu \mathrm{m} \mathrm{s}^{-1}$ (for $W=27 \mathrm{dBm}$ ) to $2.6 \mathrm{~mm} \mathrm{~s}^{-1}$ (for $W=30 \mathrm{dBm}$ ), corresponding to Reynolds numbers (Re) of $10^{-3}-10^{-2}$. Therefore, upon doubling the operation power, a penetration speed more than 20 times larger is found at the end of our microchannels. The corresponding average pumping rates $(Q)$ are between 0.05 and $0.2 \mathrm{nl} \mathrm{s}^{-1}$ (Fig. 8). Experiments on microchannels with cross section up to $520 \times 50 \mu \mathrm{m}^{2}$ lead to similar results, with maximum registered velocities of the order of many $\mathrm{mm} \mathrm{s}^{-1}$ (Fig. 7b), and flow rates up to many tens of $\mathrm{nl} \mathrm{s}^{-1}$ (Fig. 8).

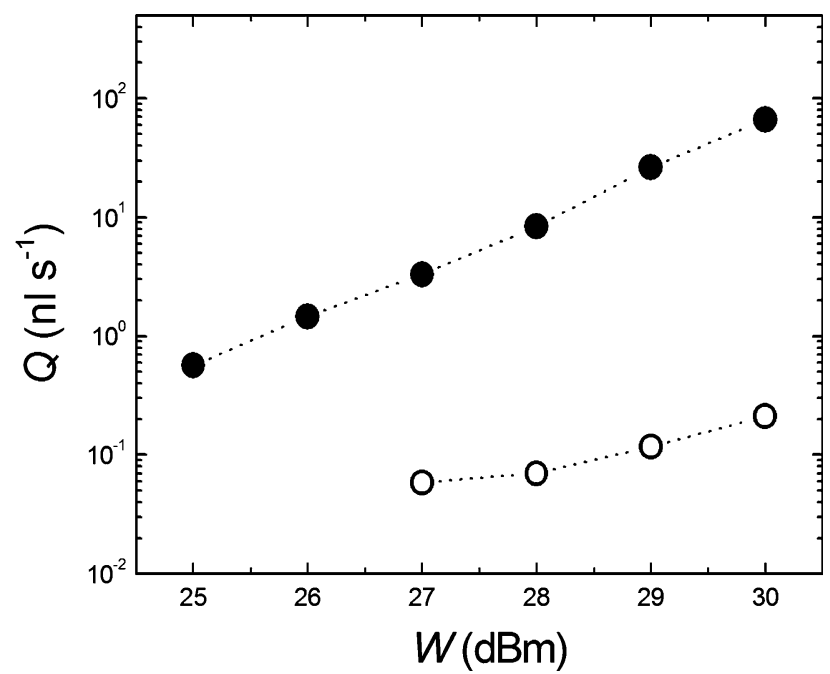

Fig. 8 Average flow rate of withdrawing micropumping in $210 \times 20 \mu \mathrm{m}^{2}$ (open circles) and $520 \times 50 \mu \mathrm{m}^{2}$ (full circles) microchannels $v s$ excitation power, $W$. Dotted lines are guides for the eye.

In a simplified phenomenological model not taking into account the acoustic agitation components along the $x$ and $y$ coordinates of the capillary, the net effect of the SAW withdrawing on the fluid can be described by an acoustic power exponentially-damped with decay length, $\lambda_{\mathrm{s}}$, upon moving away from the IDT along the microchannel. The penetration coordinate, $z$, is then described by:

$$
\mathrm{d} z / \mathrm{d} t=\left(R^{2} / 8 \eta\right)\left[\left(P_{\mathrm{C}} / z\right)+\left(P_{\mathrm{S}} / \lambda_{\mathrm{s}}\right) \exp \left(z / \lambda_{\mathrm{s}}\right)\right]
$$

In the previous equation, $R$ is hydraulic radius of the capillary, $P_{\mathrm{S}}$ indicates the acoustic power at the entrance of the microchannel from where water is sucked, and the capillarity pressure $\left(P_{\mathrm{C}}\right)$ is expressed by the Young-Laplace equation, namely $P_{\mathrm{C}}=2 \sigma(\cos \theta) / R$, where $\sigma$ and $\theta$ indicate the surface tension of the liquid and its effective contact angle with respect to the channel walls, respectively. By disregarding the capillary pressure, according to which the fluid would not penetrate in the channels, with respect to the dominant acoustic component, one obtains the following relation between $z$ and $t$ :

$$
t=8 \eta \lambda_{\mathrm{s}}^{2}\left[1-\exp \left(-z / \lambda_{\mathrm{s}}\right)\right] /\left(P_{\mathrm{S}} R^{2}\right)
$$

The experimental curves of SAW withdrawing are in good agreement with eqn (2), with values of the decay lengths of 1$3 \mathrm{~mm}$ and values of the acoustic pressure $P_{\mathrm{S}}$ at the edge of the microchannel between 1.2 and $3.0 \mathrm{cPa}$ for a capillary with $R \cong 20 \mu \mathrm{m}$ (inset of Fig. 7a). Microchannels with $R \cong 45 \mu \mathrm{m}$ also provide data which are consistent with an exponential-like $z(t)$ growth such as in this model, although the curves collected employing our device geometry under SAW pumping with $W$ values $>25 \mathrm{dBm}$ are hardly fittable, because of the too high velocities in the microchannels and narrow spatial and temporal dynamics probed within $5 \mathrm{~mm}$ long capillaries. However, the experimental curve of SAW withdrawing with $W=25 \mathrm{dBm}$ can be fitted by eqn (2), with a value of the decay length of about $8 \mathrm{~mm}$ and values of the acoustic pressure $P_{\mathrm{S}}$ at the edge of the microchannel as large as $50 \mathrm{cPa}$ (fitting curve in Fig. 7b).

In general, SAW-induced pumping is due to the power leaks in the fluid, which induce a net pressure gradient, whose 
pattern depends on the specific device geometry. For instance, a distribution of the streaming pressure non-collinear to the inplane direction normal to the IDT can be obtained by suitable reflecting geometries determining different acoustic radiations over the different fractions of the liquid. ${ }^{31}$ In microchannels, the contact line pinning boundary conditions due to the polymeric walls surrounding the fluid may determine a complex acoustic radiation pattern, including exponential decays of pressures in the liquid and in the polymer, and multiple refractions and reflections at the involved interfaces. Furthermore, the wetting of the internal surfaces of the microchannel by droplets ${ }^{14}$ or by hydrophilic precursors ${ }^{32}$ given by the evaporation and recondensation of the filling fluid greatly contribute to enhance the flow significantly in the microchannels.

Finally, we test the compatibility of this pumping method with biological solutions. Efficient microchannel filling is easily obtained also for solutions of fluorescent proteins (inset of Fig. 9). For sake of example, in case of a channel with section $200 \times 20 \mu \mathrm{m}^{2}$ coupled to an IDT working at a resonance frequency of $151 \mathrm{MHz}$, a filling velocity of about $50 \mu \mathrm{m} \mathrm{s}^{-1}$ is measured (for $W=21.5 \mathrm{dBm}$ ). Important, the protein fluorescence intensity and spectral characteristics (peak wavelength, $\lambda_{\text {EIGFP }}$, and emission full width at half maximum, $\Delta \lambda_{\text {EIGFP }}$ ) are not significantly affected by the interaction with the SAW and with the hybrid channel during the entire filling process (Fig. 9). The unaltered protein fluorescence confirms the suitability of SAW withdrawing in microchannels for biological applications.

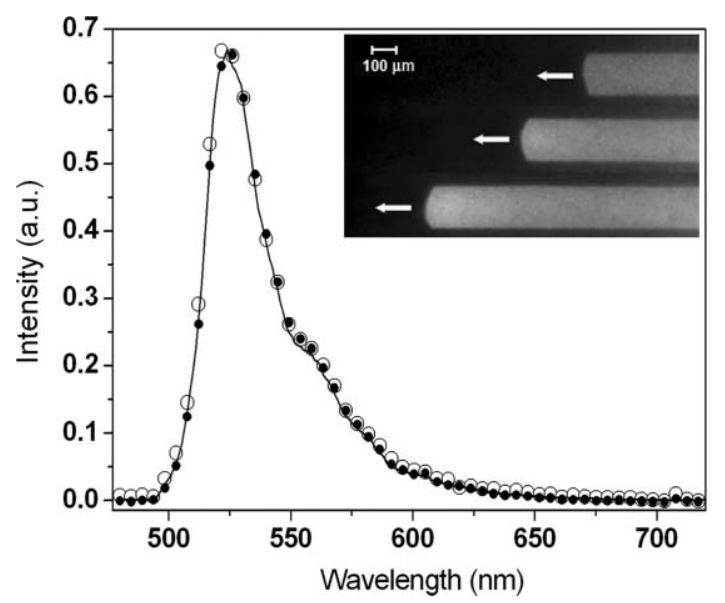

Fig. $9 E^{1}$ GFP emission spectra at the entrance of SAW microchannels (open dots), within microchannels during SAW withdrawing (solid line), and at the exit of the microchannels (full dots). No significant intensity or spectral differences are observed during and after the filling process. For all the spectra, $\lambda_{\mathrm{E} 1 \mathrm{GFP}}=524 \mathrm{~nm}$ and $\Delta \lambda_{\mathrm{EIGFP}}=$ $29 \mathrm{~nm}$. Inset: fluorescence micrographs of $\mathrm{E}^{1} \mathrm{GFP}$ solution withdrawing micropumping at different times $(t)$. From top to bottom: $t=0.00,10.15$, $19.45 \mathrm{~s}$.

\section{Conclusions}

We demonstrated prototypical microchannel devices with hybrid walls made of piezoelectric $\mathrm{LiNbO}_{3}$ and PDMS, which enable a effective control of microflow in the direction opposite to SAW propagation. In particular, the withdrawing pumping configuration was quantitatively investigated and found to be related to the SAW-induced formation and coalescence of droplets within the microchannel. The growth dynamics of single droplets in the capillary is governed by the diffusion of water on the hydrophilic $\mathrm{LiNbO}_{3}$ surface, driving to coalescence with neighbouring liquid domains or with the advancing fluid front. Observed velocities were up to $2.6 \mathrm{~mm} \mathrm{~s}^{-1}$ (for $W=30 \mathrm{dBm}$ ), corresponding to average values of pumping rates of tens of $\mathrm{nl} \mathrm{s}^{-1}$, and the SAW withdrawing was described by a model taking into account an acoustic power exponentially decaying upon travelling from the IDT along the microchannel.

Since it relies on the droplet dynamics at the air-liquid interface within the microchannels, this method needs to be applied to digital microfluidics. Once microchannels are completely full with liquids, the acoustic power is very effectively damped and hence more difficult to apply to withdrawing liquids in capillaries. However, in a number of interesting applications, digital microfluidics can be effectively coupled to microchannel environments to reduce evaporation and improve fluid control. These include, for instance, immunoassays and sensors based on the mutual contact between immobilized diagnostic probes and liquid drops carrying antigens through the chip. ${ }^{33}$

In addition, it has been noted that acoustic streaming has the remarkable advantage of scaling favourably upon decreasing the microchannel cross-section, because of the acoustic generation at the capillary boundary interfaces. ${ }^{34}$ For this reason, SAW-based withdrawing methods in hybrid microfluidic architectures certainly deserve to be fully investigated as promising approaches for flow control in many lab-on-chip applications.

\section{Acknowledgements}

The authors are grateful for the support of the Italian Institute of Technology and of the Italian Ministry of Universities and Research through the FIRB projects RBLA03ER38 and RBIN045NMB. We also thank Stefano Luin for the fluorescence measurements.

\section{Notes and references}

1 T. M. Squires and S. R. Quake, Rev. Mod. Phys., 2005, 77, 977-1026; J. Atencia and D. J. Beebe, Nature, 2005, 437, 648-655; A. K. Shaikh, K. S. Ryu, E. D. Goluch, J.-M. Nam, J. Liu, C. Shad Thaxton, T. N. Chiesl, A. E. Barron, Y. Lu, C. A. Mirkin and C. Liu, Proc. Natl. Acad. Sci. U. S. A., 2005, 102, 9745-9750; C. T. Eijkel and A. van de Berg, Microfluid. Nanofluid., 2005, 1, 249-267; P. S. Dittrich, K. Tachikawa and A. Manz, Anal. Chem., 2006, 78, 3887-3908.

2 M. Zimmermann, H. Schmid, P. Hunziker and E. Delamarche, $L a b$ Chip, 2007, 7, 119-125.

3 D. J. Laser and J. G. Santiago, J. Micromech. Microeng., 2004, 14, R35-R64; P. Woias, Sens. Actuators, B, 2005, 105, 28-38.

4 J. G. Smits, Sens. Actuators, A, 1990, 21-23, 203-206.

5 M. Stehr, S. Messner, H. Sandmaier and R. Zengerle, Sens. Actuators, A, 1996, 57, 153-157; S. Böhm, W. Olthuis and P. Bergveld, Sens. Actuators, A, 1999, 77, 223-228.

6 F. C. M. van de Pol, h. T. G. van Lintel, M. Elwenspoek and J. H. J. Fluitman, Sens. Actuators, A, 1990, 21, 198-202; O. C. Jeong and S. S. Yang, Sens. Actuators, A, 2000, 83, 249-255.

7 M. A. Unger, H. P. Chou, T. Thorsen, A. Scherer and S. R. Quake, Science, 2000, 288, 113-116; W. H. Grover, A. M. Skelley, C. N. Liu, E. T. Lagally and R. A. Mathies, Sens. Actuators, B, 2003, 89, 315-323.

8 J. Xie, J. Shih, Q. Lin, B. Yangb and Y.-C. Taia, Lab Chip, 2004, 4, 495-501. 
9 S. F. Bart, L. S. Tavrow, M. Mehregany and J. H. Lang, Sens. Actuators, A, 1990, 21, 193-197; A. Richter, A. Plettner, K. A. Hofmann and H. Sandmaier, Sens. Actuators, A, 1991, 29, 159-168; S. H. Ahn and Y. K. Kim, Sens. Actuators, A, 1998, 70, 1-5.

10 A. Homsy, S. Koster, J. C. T. Eijkel, A. Van Den Berg, F. Lucklum, E. Verpoorte and N. F. de Rooij, Lab Chip, 2005, 5, 466-471.

11 T. E. McKnight, C. T. Culbertson, S. C. Jacobson and J. M. Ramsey, Anal. Chem., 2001, 73, 4045-4049; S. Debesset, C. J. Hayden, C Dalton, J. C. T. Eijkel and A. Manz, Lab Chip, 2004, 4, 396-400; A. Brask, J. P. Kutter and H. Bruus, Lab Chip, 2005, 5, 730-738.

12 W. Satoh, H. Hosono and H. Suzuki, Anal. Chem., 2005, 77, 68576863.

13 R. H. Liu, J. Yang, R. Lenigk, J. Bonanno and P. Grodzinski, Anal. Chem., 2004, 76, 1824-1830.

14 G. L. Liu, J. Kim, Y. Lu and L. P. Lee, Nat. Mater., 2006, 5, 27-32.

15 L. Caprioli, E. Mele, F. E. Angilè, S. Girardo, A. Athanassiou, A. Camposeo, R. Cingolani and D. Pisignano, Appl. Phys. Lett., 2007, 91, 113113 .

16 M. Kurosawa, T. Watanabe, A. Futami and T. Higuchi, Sens. Actuators, A, 1995, 50, 69-74.

17 S. Shikawa, Y. Matsui and T. Ueda, Jpn. J. Appl. Phys., 1989, 28(Suppl. 28-1), 126-128; Z. Guttenberg, A. Rathgeber, S. Keller, J. O. Rädler, A. Wixforth, M. Kostur, M. Schindler and P. Talkner, Phys. Rev. E, 2004, 70, 056311.

18 Z. Guttenberg, H. Müller, H. Habermüller, A. Geisbauer, J. Pipper, J. Felbel, M. Kielpinski, J. Scriba and A. Wixforth, Lab Chip, 2005, 5, 308-317.

19 K. Sritharan, C. J. Strobl, M. F. Schneider, A. Wixforth and Z. Guttemberg, Appl. Phys. Lett., 2006, 88, 054102.

20 A. Renaudin, P. Tabourier, V. Zhang, J. C. Camart and C. Druon, Sens. Actuators, B, 2006, 113, 389-397; J. Bennès, S. Alzuaga, F. Chérioux, S. Ballandras, P. Vairac, J.-F. Manceau and F. Bastien, IEEE Trans. Ultrason. Ferroelectr. Freq. Contr., 2007, 54, 21462151.

21 D. Beyssen, L. Le Brizoual, O. Elmazria and P. Alnot, Sens. Actuators, B, 2006, 118, 380-385.
22 S. W. Schneider, S. Nuschele, A. Wixforth, C. Gorzelanny, A. Alexander-Katz, R. R. Netz and M. F. Schneider, Proc. Natl. Acad. Sci. U. S. A., 2007, 104, 7899-7903.

23 M. K. Tan, J. R. Friend and L. Y. Yeo, Lab Chip, 2007, 7, 618-625.

24 S. M. Hagsäter, T. Glasdam Jensen, H. Bruus and J. P. Kutter, Lab Chip, 2007, 7, 1336-1344.

25 Y. Xia Y. and G. M. Withesides, Angew. Chem., Int. Ed., 1998, 37, $550-575$.

26 S. Pagliara, L. Persano, A. Camposeo, R. Cingolani and D. Pisignano, Nanotechnology, 2007, 18, 175302.

27 R. Bizzarri, R. Nifosi, S. Abbruzzetti, W. Rocchia, S. Guidi, D. Arosio, G. Garau, B. Campanini, E. Grandi, F. Ricci, C. Viappiani and F. Beltram, Biochemistry, 2007, 46, 5494; D. Arosio, G. Garau, F. Ricci, L. Marchetti, R. Bizzarri, R. Nifosi and F. Beltram, Biophys. J., 2007, 93, 232.

28 For our microchannel geometry, embedding one (hydrophilic) $\mathrm{LiNbO}_{3}$ and three (hydrophobic) PDMS surfaces, the wettability is different along the different capillary walls (with liquid-surface contact angles $\theta_{\mathrm{LiNbO}_{3}}$ and $\theta_{\text {PDMS }}$, respectively (Fig. $3 \mathrm{~g}$ and $\mathrm{h}$ ). The corresponding form of the capillarity pressure can be brought to the Young-Laplace form by defining a suitable effective contact angle and the hydraulic radius as twice the height of the microchannel.

29 M. Cecchini, S. Girardo, D. Pisignano, R. Cingolani and F. Beltram, Appl. Phys. Lett., 2008, 92, 104103; R. Cingolani, M. Cecchini, F. Beltram, S. Girardo and D. Pisignano, Italian Patent Application, TO2007A000554.

30 T. M. Rogers, K. R. Elder and R. C. Desai, Phys. Rev. A, 1988, 10, 5303-5309.

31 H. Li, J. R. Friend and L. Y. Yeo, Biomed. Microdevices, 2007, 9 , 647-656.

32 D. I. Dimitrov, A. Milchev and K. Binder, Phys. Rev. Lett., 2007, 99, 054501.

33 A. Bange, H. Brian Halsall and W. R. Heineman, Biosens. Bioelectron., 2005, 20, 2488.

34 K. D. Frampton, K. Minor and S. Martin, Appl. Acoust., 2004, 65, 1121-1129. 